\title{
Fading Correlation Analysis in MIMO-OFDM Troposcatter Communications: Space, Frequency, Angle and Space-frequency Diversity
}

\author{
Ergin Dinc, Student Member, IEEE, Ozgur B. Akan, Senior Member, IEEE
}

\begin{abstract}
The capacity gain of MIMO systems significantly depends on the fading correlation between antennas, and there is no analytical study which considers the fading correlation in the troposcatter communications. In this paper, we develop an analytical model, ring scatter model (RSM), to derive the fading correlation in the troposcatter systems as a function of spatial, frequency and angular separations for the first time in the literature. In addition, we compare the effects of the diversity techniques that are suitable for troposcatter communications: space, frequency, angle and space-frequency diversity techniques by deriving the distribution of their achievable data rates with transmit beam-forming. To this end, we extend our previously introduced troposcatter channel model [1] for the implementation of MIMO-OFDM and the diversity techniques.
\end{abstract}

Index Terms-Electromagnetic scattering, Correlation, Communication channels, Diversity methods, MIMO systems

\section{INTRODUCTION}

$\mathbf{T}$ ROPOSCATTER is the scattering of the propagating signals due to atmospheric irregularities. Although most of the scattered power is directed to the forward direction, it is possible to receive some of the scattered power at the receiver by pointing the antennas to the horizon as can be seen from Figure 1. The intersection of the antenna beam-widths are denoted as troposcatter common volume and the receiver can receive the scattered rays only in this region. Therefore, troposcatter can be used as a communication medium for high data rate beyond-Line-of-Sight (b-LoS) communications [1].

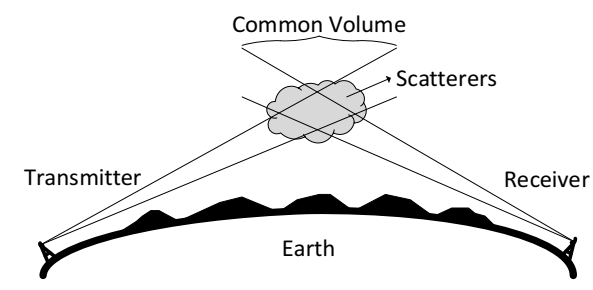

Fig. 1. Troposcatter b-LoS paths.

Troposcatter communication is a promising candidate for b-LoS communications with its low transmission delays and high capacity. The available b-LoS communications mostly utilize satellite communications (SATCOM) or relay nodes. SATCOM has excessive transmission delays and capacity

E. Dinc and O. B. Akan are with the Next-generation and Wireless Communications Laboratory, Department of Electrical and Electronics Engineering, Koc University, Istanbul, 34450 Turkey (e-mail: edinc@ku.edu.tr; akan@ku.edu.tr). problems under low coverage. In addition, the employment of relay nodes has security problems due to possible hostile attacks. Since the troposcatter communication provides a direct communication link with microsecond transmission delays [2], the modern b-LoS communication requiring high data rates can utilize the troposcatter communications.

Troposcatter medium is a lossy wave-guide due to high path lengths and scattering. Thus, the implementation of diversity techniques is required to provide reliable and high data rate b-LoS troposcatter systems. The main diversity techniques for troposcatter communications are space, frequency and angle diversity. In addition, the combination of diversity techniques may be utilized to increase the spectral efficiency with less cost. For example, the space-frequency diversity employs horizontally placed antennas utilizing frequency diversity. Since the frequency diversity receivers can be mounted on the same parabolic reflector, quad diversity can be achieved with only 2 antennas in each side. Therefore, the main objective of this paper is to analyze the correlation between antennas in these diversity techniques to determine the proper spacings to achieve the desired gains for the first time in the literature.

The contribution of this paper is twofold: Firstly, we develop an analytical model for the fading correlation of the troposcatter antennas utilizing space, frequency and angle and space-frequency diversity techniques for the first time. These diversity techniques are employed by the modern troposcatter systems [2], but there are no analytical fading correlation studies. Therefore, we develop the Ring Scatter Model (RSM) to analyze the fading correlation in troposcatter links and compare our results with the empirical values provided by the industry [2], ITU [3], and the experimental results [4]-[9]. In addition, one of the most important contributions of this work is the prediction of $50 \%$ coherence bandwidth based on the frequency separation results. In this way, the fading behavior of the channel can be determined for a given channel parameters. Secondly, we compare the employment of different diversity techniques with the distribution of achievable data rates by extending our previously proposed ray-based channel model approach for troposcatter communications [1]. Our raybased channel model is a ray-tracing method which calculates the power and delay of each ray to generate power delay profile (PDP). The novelty of our approach is to use the real world measurements for atmospheric modeling, thus each ray is subjected to different conditions according to its height (For more details, see [1]). In this work, we extend our approach to implement the diversity techniques and MIMO-OFDM. 
The reminder of the paper is organized as follows. Section II presents the related work. Section III provides the RSM fading model. In Section IV, the ray-based channel model is reviewed. Section V and VI include the formulation and simulation results for the data rates for the diversity techniques, respectively. The conclusions are presented in Section VII.

\section{RELATED WORK}

Although the fading correlation is well studied especially in mobile links [10], [11], [13], there is no theoretical study which considers the fading correlation in the troposcatter links. Therefore, our main aim is to analyze the fading correlation in different diversity schemes: space, frequency, angle, and space-frequency diversity techniques. On the other hand, there are empirical recommendations for these diversity techniques in order for the antennas to be uncorrelated [2], [3]. In addition, there are available experimental studies which considers the fading correlation in the troposcatter links. [6], [7] provides the fading correlation results for space diversity along with the fading statistic of the channel. According to their results, the channel shows Rayleigh fading under low integration times. [4], [5] focus on frequency correlation in the troposcatter links. The correlation and path-loss results for the angle diversity systems are provided in [8], [9]. We utilize these empirical suggestions and experimental studies to to validate our results.

To model the fading correlation, there are several methods. For this purpose, the fading correlation can be determined by channel sounding experiments [10]. However, the cost of channel sounding experiments for troposcatter is too high due to the requirement for high power amplifiers and large antennas. Therefore, the correlation between antennas may be determined via analytical studies. This is the main reason for this paper. In addition, one of the most popular fading correlation model is "One Ring" model, which is first introduced in [13]. In this model, the receiver is assumed to be surrounded by local scatterers and the correlation between the antennas are determined by using channel geometry calculations. However, the scatterers in troposcatter channel are located in the troposcatter common volume as in Figure 1 unlike the one ring model. Therefore, we develop the RSM for the fading correlation in the troposcatter system based on the methodology in [11], [13] by using the troposcatter channel geometry [14]. We extend RSM to analyze space, frequency, angle and space-frequency diversity fading correlation calculations.

\section{Ring SCATter Model For Troposcatter FAding CORRELATIONS}

To achieve the desired gains with MIMO, the antennas should have desired correlation values. Thus, we develop an analytical model, ring scatter model (RSM), to investigate the fading correlations in the main diversity techniques that are suitable for the b-LoS troposcatter communications: space, frequency and angle diversity. In addition, the combination of diversity techniques also has a potential to provide higher achievable data rates. Therefore, space-frequency diversity which is the combination of space and frequency diversity is introduced and investigated as well.
RSM fading correlation analysis are developed for singleuser to single-user communication link. Troposcatter link has $M_{t, r}$ antennas at the transmitter and receiver sides, respectively. Therefore, the channel response in frequency domain $\left(\mathbf{H}_{M_{r} \times M_{t}}[k]\right)$ can be represented as

$$
\mathbf{r}[k]=\mathbf{H}[k] \mathbf{s}[k]+\mathbf{v}[k],
$$

where $\mathbf{r}_{M_{r} \times 1}$ is the received signal vector, $\mathbf{s}_{M_{t} \times 1}$ represents the transmit vector, $\mathbf{v}_{M_{r} \times 1}$ is additive-white-Gaussian-noise (AWGN).

The remainder of the section includes the scattering model, and the RSM method for the diversity techniques.

\section{A. Scattering Model}

The troposcatter power depends on both the path geometry and the atmospheric turbulence. Therefore, both of these factors will have strong effects on the correlation analysis. The troposcatter is caused by the atmospheric scintillations due the the changes in the refractive index of the atmosphere. According to the turbulence characteristics, the scattering can be modeled as single or multiple scattering [16], [27]. The microwave propagation in the troposphere is related to the tenuous distribution of the particles, and it can be modeled with the first order multi-scattering approximation in the tenuous medium for a unit particle as [16], [27]

$$
\frac{P_{r}}{P_{t}}=\frac{\lambda^{2} G_{t}(\hat{i}) G_{r}(\hat{o})}{(4 \pi)^{3} R_{t}^{2} R_{r}^{2}} \sigma_{V}(\hat{i}, \hat{o}, n) \exp \left(-\tau_{t}-\tau_{r}\right),
$$

where $\lambda$ is the wavelength, $P_{t, r}$ are the transmitter and receiver power. $G_{t, r}$ are the antenna gains which are modeled with Gaussian pattern. $R_{t, r}$ are the distances between scattering point to transmitter and receiver respectively. $\sigma_{V}(\hat{i}, \hat{o}, n)$ is the scattering cross-section, where $\hat{i}$ is the incoming ray vector, $\hat{o}$ is the scattered ray vector, and $n$ is the index of refraction. $\tau_{t, r}$ are the optical distances for $R_{t, r}$ [16].

In correlation calculations, the constant terms can be omitted to simplify the model because they will have no effect: $\lambda^{2}$ and $(4 \pi)^{3}$ in (2). In addition, the terms that have very slight changes can be eliminated. $R_{t}^{2} R_{r}^{2}$ term in of (2) will also show very slight changes due to narrow beam-widths. For $250 \mathrm{~km}$ range and $1.5^{\circ}$ beam-width, the ratio of the minimum path over maximum path $\left(R_{t}^{M i n} / R_{t}^{M a x}\right)$ is found as 0.9992 . Thus, this term can be eliminated to simplify the model. Furthermore, the $\tau_{t, r}$ are the path integral of the $R_{t}$ and $R_{r}$ over the index of refractions and and they represents the effect of the scattering particles between the antennas and the common volume. Since the expected antenna spacing in space diversity $(\approx 100 \lambda \ll$ $R$ ), and no spacing for the frequency and angle diversity, the values for optical distances will be almost the same for each ray. Therefore, we eliminate the $\exp \left(-\tau_{t}-\tau_{r}\right)$ in (2) as well.

To model the atmospheric turbulence, we use BookerGordon model [16] instead of the Kolmogorov theory which is utilized in [1] because the correlation within the atmospheric turbulence can be directly modeled with BookerGordon model. In this model, the correlation function of the atmospheric scintillations is modeled with the exponential function, and it is given as [16]

$$
B_{n}\left(l_{x}, l_{y}\right) \approx \exp \left(-l_{x} / l_{x}^{c}-l_{y} / l_{y}^{c}\right),
$$




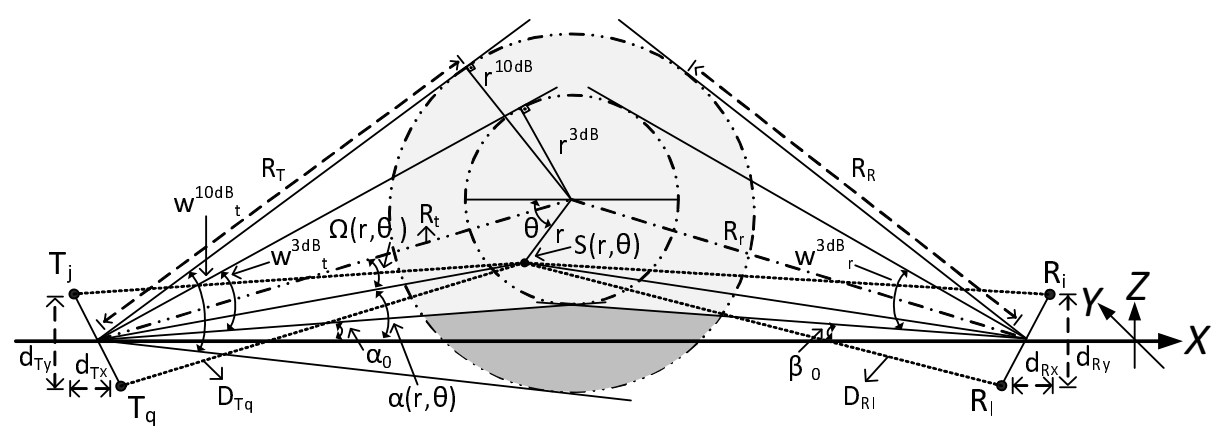

Fig. 2. Geometry of RSM method.

where $l_{x, y}$ are the horizontal and vertical distance to the reference point, and $l_{x, y}^{c}$ are the correlation distances of the turbulence for vertical and horizontal axis. For troposcatter, $l_{y}^{c}$ is approximately $\approx 50 \mathrm{~m}$ and it varies $20-130 \mathrm{~m}$ according to the atmospheric conditions [16]. Since $l_{x}^{c}$ is much greater than $l_{y}^{c}$ as in [16], we assume that $l_{x}=4 \times l_{y}$ [16].

The geometry dependency of the scattering cross-section can be determined with $\sin (\Psi)^{-11 / 3}$ as experimentally validated in [9]. With the proposed simplifications for the correlation analysis, the resulting power relationship in the troposcatter channel is found as

$$
P_{r} / P_{t} \approx \sin (\Psi)^{-11 / 3} B_{n}\left(l_{x}, l_{y}\right) G_{t}(\hat{i}) G_{r}(\hat{o}),
$$

where $D_{T_{j}}$ is the distance between scatterer $(S(r, \theta))$ and transmitter $j$, and $D_{R_{i}}$ is the distance between scatterer $S(r, \theta)$ and receiver $i$ as in Figure 2. Since the antenna gains will change dramatically for $10 \mathrm{~dB}$ beam-widths, we include the antenna gains in the model as well.

The scattering model which is considered in this paper is developed for the clear air atmospheric turbulence between 1$10 \mathrm{GHz}$ because the atmospheric correlation model is valid for the clear air conditions and the scattering power relationship depends on the Rayleigh scattering which is more accurate for $1-10 \mathrm{GHz}$ [16]. In addition, we assume that the transmitter and receiver have the same vertical orientation for simplicity. Therefore, we consider only the symmetrical troposcatter paths in this paper. However, our model can be extended for nonsymmetrical cases with geometrical approximations.

\section{B. RSM Model}

In troposcatter, only the scattered rays inside the troposcatter common volume can be received due to path geometry as in Figure 1. The scattered rays outside of this region will reach the receiver with either lower or higher angles than the $10 \mathrm{~dB}$ beam-width of the receiver. Although the scattering particles are located through the troposphere, we only consider the scatterers that are located in a ring within the intersection of the transmitter and receiver $10 \mathrm{~dB}$ beam-widths as in Figure 2. The boundaries for the scatterers is given as $\theta$ in $[-\pi, \pi)$ and $r$ in $\left[0, r^{10 d B}\right]$. The radius of the rings are given by

$$
\begin{aligned}
r^{3 d B} & =R_{t} \sin \left(w_{t}^{3 d B} / 2\right), \\
r^{10 d B} & =R_{t} \sin \left(w_{t}^{10 d B} / 2\right),
\end{aligned}
$$

where $R_{t}$ is the path between the center of the rings and transmitter. $w_{t}^{3 d B}$ and $w_{t}^{10 d B}$ are the transmitter $3 \mathrm{~dB}$ and $10 \mathrm{~dB}$ beam-widths, respectively.

To maximize the received power, the $3 \mathrm{~dB}$ beam-widths of the antennas are adjusted to the radio horizon as in Figure 2 [1], [14]. Therefore, the lower part of the ring (the darker region in Figure 2) will be blocked by the path geometry due to the curvature of the earth. Since RSM method utilizes the 10 $\mathrm{dB}$ beam-widths, the lower part of the ring will be eliminated from the correlation calculations as described in Appendix A.

Due to the troposcatter geometry, the path-loss observed by the both end of the communication is symmetrical [2]. The troposcatter links generally utilize the same type of antennas at the both ends. For these reasons, we assume that the troposcatter path is symmetrical. Thus, we have $w_{t}^{3 d B}=w_{r}^{3 d B}$ and $w_{t}^{10 d B}=w_{r}^{10 d B}$ that imply $R_{T}=R_{R}$ as [1]

$$
R_{T, R}=R \sin \left(\beta_{0}\right) / \sin \left(\alpha_{0}+\beta_{0}\right),
$$

where $\alpha_{0}$ and $\beta_{0}$ are the elevations of the antenna beams with respect to horizon for transmitter and receiver, respectively [1]. $R$ is the horizontal distance between transmitter and receiver.

Since the b-LoS troposcatter communication does not have any line-of-sight component, only the scattered rays are considered in RSM and the power of the scattered rays are determined by (4). Suppose that there are $K$ effective scatterers in each ring and $L$ rings within the common volume. Thus, the normalized path gain $\mathbf{H}_{i, j}$ is represented as [11]

$$
\begin{gathered}
\mathbf{H}_{i, j}=\int_{0}^{r^{10 d B}} \int_{0}^{2 \pi} \sum_{n=1}^{L} \sum_{k=1}^{K} \delta\left(\theta-\theta_{k}\right) \delta\left(r-r_{n}\right) \zeta(r, \theta) \\
G_{t}(r, \theta) G_{r}(r, \theta) \sin (\Psi(r, \theta))^{-11 / 3} B_{n}\left(l_{x}, l_{y}\right) \\
\quad \exp \left(-j 2 \pi / \lambda\left(D_{T_{j}}+D_{R_{i}}\right)+j \phi(r, \theta)\right) d \theta d r,
\end{gathered}
$$

$\zeta(r, \theta)$ is defined to exclude the lower part of the ring as described in Appendix A. $G_{t, r}(r, \theta)$ are the antenna gains, as defined in Appendix B. In Section III-A, the multi-scatter nature of the troposcatter is approximated by the single scattering [16]. Therefore, the phase $(\phi(r, \theta))$ is assumed as uniformly distributed $[-\pi, \pi)$ and iid. Thus, phase term is canceled in the correlation analysis as in [11].

For the fading correlation analysis, $\mathbf{H}_{M_{r} \times M_{t}}$ matrix is reshaped into $\widehat{\mathbf{h}}_{M_{r} M_{t} \times 1}$ vector. In this case, the fading correlation between different antenna pairs becomes the covariance 


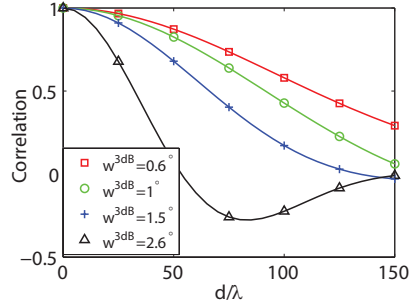

(a)

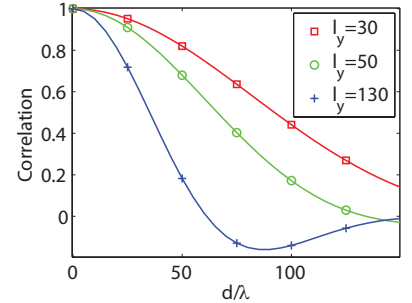

(b)

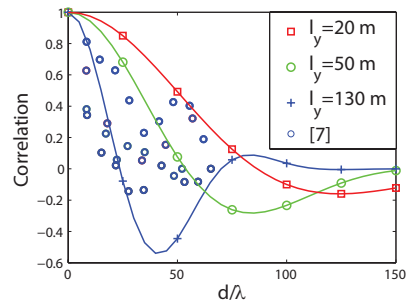

(c)

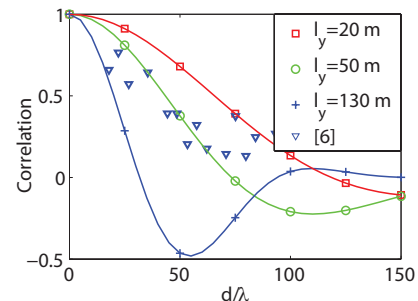

(d)

Fig. 3. Fading correlation vs. spatial separation (a) for different beam-widths, and (b) for different correlation distance, (c) and (d) for the comparison with the experimental measurements.

between $\widehat{\mathbf{h}}_{i, j}$ and $\widehat{\mathbf{h}}_{l, q}$ that can be represented as $E\left[\widehat{\mathbf{h}}_{i, j} \widehat{\mathbf{h}}_{l, q}^{*}\right]$ where $\left(.^{*}\right)$ is the conjugate operation [11].

\section{Space Diversity}

We assume that there are infinitely many scatterers inside the scattering common volume, thus $L$ and $K$ in (7) go to infinity. By using (7), the spatial fading correlation between different troposcatter antenna pairs is represented as

$$
\begin{aligned}
E\left[\widehat{\mathbf{h}}_{i, j} \widehat{\mathbf{h}}_{l, q}^{*}\right]= & \int_{0}^{r^{10 d B}} \int_{0}^{2 \pi} B_{n}(|r \sin (\theta)|,|r \cos (\theta)|)^{2} \\
& G_{t}(r, \theta) G_{r}(r, \theta) \zeta(r, \theta) \xi(r, \theta) \\
& \exp \left(-j 2 \pi / \lambda\left(\Delta D_{T_{j, q}}+\Delta D_{R_{i, l}}\right)\right) d \theta d r
\end{aligned}
$$

where $\Delta D_{T_{j, q}}=D_{T_{j}}-D_{T_{q}}$ is the path length difference between local scatterer to transmitters $j$ and $q$, and $\Delta D_{R_{i, l}}=D_{R_{i}}-D_{R_{l}}$ is the path length difference between local scatterer to receivers $i$ and $l . \xi(r, \theta)$ function represents the $\sin \left(\Psi_{1}\right)^{-11 / 3} \times \sin \left(\Psi_{2}\right)^{-11 / 3}$ term as a function of $r$ and $\theta$ as defined in Appendix C. $B_{n}$ is the correlation function (3). Since the reference point is assumed as the center of the ring, the vertical and horizontal distance from the reference can be simply find by $|r \sin (\theta)|$ and $|r \cos (\theta)|$, respectively.

Since troposcatter paths use low beam-widths and elevation angles, (8) can be simplified by using geometrical approximations. For low beam-widths, $\Delta D_{T_{j, q}} \approx d_{T_{x}} \cos (\Omega(r, \theta))+$ $d_{T_{y}} \sin (\Omega(r, \theta))$, where $\sin (\Omega(r, \theta)) \approx r / R_{t} \sin (\theta)$, and $\cos (\Omega(r, \theta)) \approx\left(1-\frac{1}{4}\left(r / R_{t}\right)^{2}+\frac{1}{4}\left(r / R_{t}\right)^{2} \cos (2 \theta)\right)=$ $\xi(r, \theta)$ where $d_{T_{x, y}}$ are the vertical and horizontal spacing of the transmitters. $R_{t}$ is the distance between transmitter and the center of the troposcatter common volume as in Figure 2. Since the troposcatter path is assumed as symmetrical, $R_{t}=R_{r}$ and given as

$$
R_{t, r}=R \frac{\sin \left(\beta_{0}+w_{t}^{3 d B} / 2\right)}{\sin \left(\alpha_{0}+\beta_{0}+w_{t}^{3 d B}\right)} .
$$

By using the geometrical approximations, $\Delta D_{T_{j, q}}+\Delta D_{R_{i, l}}$ term in (8) is represented as

$$
\begin{aligned}
\Delta D_{T_{j, q}}+\Delta D_{R_{i, l}}= & d_{T_{y}} r / R_{t} \sin (\theta)+d_{T_{x}} \xi(r, \theta)+ \\
& +d_{R_{y}} r / R_{r} \sin (\theta)+d_{R_{x}} \xi(r, \theta) .
\end{aligned}
$$

We only consider horizontal space diversity because it is the most common type of spatial diversity for troposcatter. Therefore, $x$ axis component of the receive and transmit antennas becomes $d_{T_{x}}=d_{R_{x}}=0$. Since the troposcatter channel is symmetrical for transmit and receive sides, the correlation calculations that are developed for one side will be valid for the other side as well. For this reason, we only focus on the correlation between two receivers which are communicating with the same transmitter. This condition implies that $d_{T_{y}}=0$. By using (8), (10) and geometrical approximations, the correlation for horizontally spaced receivers is given as

$$
\begin{gathered}
E\left[\widehat{\mathbf{h}}_{i, j} \widehat{\mathbf{h}}_{i, l}^{*}\right]=\int_{0}^{r^{10 d B}} \int_{0}^{2 \pi} \xi(r, \theta) B_{n}(|r \sin (\theta \mid),| r \cos (\theta) \mid)^{2} \\
\zeta(r, \theta) G_{t}(r, \theta) G_{r}(r, \theta) \exp \left(-j 2 \pi / \lambda d_{R_{y}} r / R_{t} \sin (\theta)\right) d \theta d r .
\end{gathered}
$$

(11) can be solved with numerical integration on MATLAB. The calculated correlation results are normalized based on the zero spacing value which has the correlation of 1 .

Figure 3(a) shows the correlation vs. antenna spacing to wavelength ratio $(d / \lambda)$ by (11) for $250 \mathrm{~km}$ range and $l_{y}^{c}=50$ $\mathrm{m}$. As noticed, the increase in the beam-width decreases the correlation at the same ratio. The increase in the beam-width increases $r_{10 d B}$ by virtue of (5), and this condition also increases the troposcatter common volume. As the common volume increase, the separations between scatterers have higher distances and less correlation. Figure 3(b) shows the spatial correlation results for different vertical correlation distances for $1.5^{\circ} 3 \mathrm{~dB}$ beam-widths. As noticed, there is a inverse relationship between the correlation distance and correlation.

According to [2], the antennas should be placed at least $100 \lambda$ or higher distance apart to achieve low correlation values. Our results in Figure 3(a) also show that $100 \lambda$ spacing is required for $1^{\circ}$ and $1.5^{\circ}$ beam-widths to achieve low correlation values. Since $4.7 \mathrm{GHz}$ troposcatter system with 3 $\mathrm{m}$ antenna diameter has $\approx 1.5^{\circ}$ beam-width $\left(w=\frac{70 \lambda}{D_{a n t}}\right.$ where $D_{\text {ant }}$ is the antenna diameter [12]), we can conclude that the our analytical results are consistent with the empirical results.

In addition, Figure 3(c) and 3(d) provides the comparison of the RSM results with the experimental results for $258 \mathrm{~km}$ and $2.6^{\circ} 3 \mathrm{~dB}$ beam-width (Figure 3 in [7]), and for $321 \mathrm{~km}$ and $2.13^{\circ} 3 \mathrm{~dB}$ beam-width (Figure 7 in [6]). As noticed, our correlation results with the same channel parameters in Figure 3(c) and 3(d) are consistent with the experimental results presented in [6], [7]. However, some deviations between the theoretical and experimental results can be observed in these figures. Since the RSM considers the clear air propagation, the 
real world atmospheric conditions may causes changes in the correlation values. On the other hand, the general trend of the correlations can be well-estimated with RSM.

\section{Frequency Diversity}

For frequency diversity, the effect of the frequency separation is reflected in the system with the small change in wavelength $(\lambda)$ in (7). In this way, the wavelength for antenna pairs will slightly differ. Therefore, the resulting covariance of (7) for antennas having frequency separation is represented as

$$
\begin{gathered}
E\left[\widehat{\mathbf{h}}_{i, j} \widehat{\mathbf{h}}_{l, q}^{*}\right]=\int_{0}^{r^{10 d B}} \int_{0}^{2 \pi} G_{t}(r, \theta) G_{r}(r, \theta) \xi(r, \theta) \\
\zeta(r, \theta) B_{n}(|r \sin (\theta \mid),| r \cos (\theta) \mid)^{2} \\
\exp \left\{-j 2 \pi\left(\frac{D_{T_{j}}+D_{R_{i}}}{\lambda_{j}}-\frac{D_{T_{q}}+D_{R_{l}}}{\lambda_{q}}\right)\right\} d \theta d r,
\end{gathered}
$$

where $\lambda_{j}=c /(f+\Delta f), \lambda_{q}=c / f$, and $\Delta f$ is the frequency separation. Therefore, the path calculations in (12) becomes

$$
\begin{aligned}
& \left(D_{T_{j}}+D_{R_{i}}\right)(f+\Delta f) / c-\left(D_{T_{q}}+D_{R_{l}}\right) f / c= \\
& \left(D_{T_{j}}+D_{R_{i}}\right) \Delta f / c+\left(\Delta D_{T_{j, q}}+\Delta D_{R_{i, l}}\right) f / c .
\end{aligned}
$$

Firstly, we assume there is no spatial separation between receivers to investigate the effects of frequency diversity. Therefore, $\left(\Delta D_{T_{j, q}}+\Delta D_{R_{i, l}}\right) f / c$ term in (13) disappears. $D_{T_{j}}$ and $D_{R_{i}}$ are found by using their relationship with $R_{r}$. Since the distance between antennas and scattering common volume is much higher than the radius of common volume $\left(R_{t, r} \gg r^{10 d B}\right)$, we can use far distance approximation in which $R_{t}$ and $D_{T_{j}}$ are assumed to be parallel to each other. In the same way, $R_{r}$ and $D_{R_{i}}$ also become parallel as shown in Figure 4. By performing the required geometrical calculations, the $D_{T_{j}}$ and $D_{R_{i}}$ is represented as

$$
\begin{aligned}
& D_{T_{j}}=R_{t}-r \cos (\theta-\alpha), \\
& D_{R_{i}}=R_{r}+r \cos (\theta+\beta) .
\end{aligned}
$$

The proof of these equations are left to the reader. Since the troposcatter path is assumed as symmetrical, $R_{t}=R_{r}$. Thus, $D_{T_{j}}+D_{R_{i}}$ term becomes

$$
D_{T_{j}}+D_{R_{i}}=2 R_{r}-r \cos (\theta-\alpha)+r \cos (\theta+\beta) .
$$

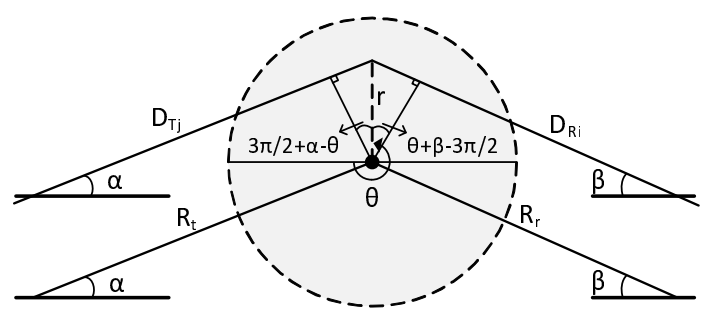

Fig. 4. Channel geometry for the frequency diversity.
By using (12), (13) and (16), the covariance expression for frequency diversity is given as

$$
\begin{array}{r}
E\left[\widehat{\mathbf{h}}_{i, j} \widehat{\mathbf{h}}_{l, q}^{*}\right]=\int_{0}^{r^{10 d B}} \int_{0}^{2 \pi} \xi(r, \theta) \zeta(r, \theta) G_{t}(r, \theta) G_{r}(r, \theta) \\
B_{n}(|r \sin (\theta \mid),| r \cos (\theta) \mid)^{2} \exp \{-j 2 \pi(\Delta f / c \\
\left.\left.\left(2 R_{r}-r \cos (\theta-\alpha)+r \cos (\theta+\beta)\right)\right)\right\} d \theta d r .
\end{array}
$$

Figure 5(a) presents the fading correlation vs. the frequency separation for different beam-widths in a $250 \mathrm{~km}$ and correlation distance $50 \mathrm{~m}$ troposcatter link. As in the space diversity, the increase in the beam-width decreases the correlation due to the increase in the common volume. According to the empirical recommendations provided by ITU [3], the frequency spacing between antennas $(\Delta f)$ should be $\Delta f=1.44 f\left(D_{\text {ant }}^{2}+15^{2}\right)^{1 / 2} / \theta R \mathrm{Mhz}$, where $f$ is the carrier frequency in $\mathrm{Hz}$ and $R$ is the range in $\mathrm{m}$. For 4.7 $\mathrm{GHz}$ carrier frequency, $3 \mathrm{~m}$ antenna diameter and $250 \mathrm{~km}$ range, the recommended frequency spacing is $13 \mathrm{MHz}$. For the same antenna diameter $\left(1.5^{\circ}\right.$ curve in Figure 5(a)), 10 $\mathrm{MHz}$ and greater frequency separations allow the antennas to be uncorrelated in the frequency diversity systems.

In addition, [4], [5] provides experimental frequency correlation results for the troposcatter links. To this end, Figure 5(c) provides the experimental frequency correlation results (Figure 9 in [5]) along with the RSM results by using the experimental channel parameters: $192 \mathrm{~km}$ and $1.17^{\circ} 3 \mathrm{~dB}$ beam-width at 7.6 GHz. As noticed, the trend of the experimental and RSM results are similar. However, the results of the RSM model is higher than the experimental results. This situation can be caused by the atmospheric conditions of the experiment site. However, the behavior of the frequency correlation can be well-captured with the RSM method.

The frequency separation formula (17) depends on the range by (9) as in the ITU's formula [3]. Figure 5(b) presents the fading correlation vs. range and frequency separation with the RSM for $1.5^{\circ} 3 \mathrm{~dB}$ beam-width and $50 \mathrm{~m}$ vertical correlation distance. According to our results, the increasing range decreases the fading correlation at the same frequency separation value because the increasing range also cause increase in the troposcatter common volume. Also, the ITU's frequency separation formula is inversely proportional to range.

Most importantly, the analytical fading calculations for the frequency diversity can be utilized to derive the coherence bandwidth of the channel for $50 \%$ correlation. According to our results, $250 \mathrm{~km}$ troposcatter link at $4.7 \mathrm{GHz}$ and $3 \mathrm{~m}$ antenna diameter have $B_{c}(50) \approx 2 \mathrm{MHz}$. Therefore, the channel will have frequency-selective fading for bandwidths greater than $2 \mathrm{MHz}$. In such cases, the channel requires the implementation of orthogonal-frequency-division-multiplexing (OFDM) to mitigate the effects of frequency-selective fading. In this paper, we focus on the high data rate employments of troposcatter communications. Thus, we use higher bandwidths than the coherence bandwidth and introduce OFDM capacity calculations for the troposcatter systems by using our previously developed ray-based method [1] in Section V. 


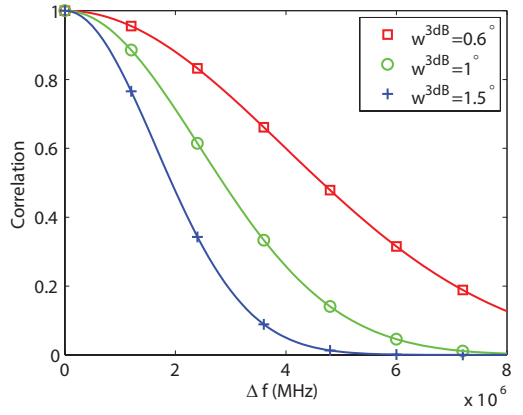

(a)

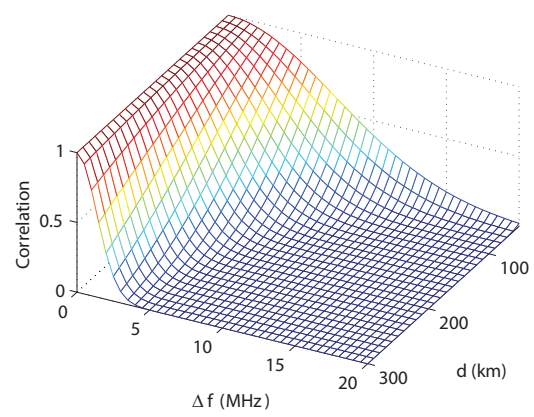

(b)

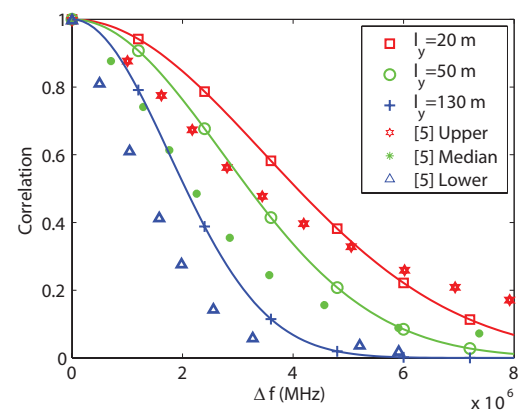

(c)

Fig. 5. The fading correlation results for (a) frequency separation, (b) range and frequency separation.

\section{E. Space-frequency Diversity}

Troposcatter systems can also use the combination of diversity techniques. Space-frequency diversity is a promising technique which can provide higher data rates with low cost. Space-frequency diversity systems utilize horizontally placed antennas with frequency diversity. Since the frequency diversity antennas can be mounted on the same parabolic reflector antenna, higher gains can be achieved with the spacefrequency diversity by using the same number of parabolic reflector antennas as in space diversity. Therefore, we derive analytical expressions for the correlation between antennas as a function of spatial and frequency separation.

By using (12) and (13), the fading correlation for spacefrequency diversity can be found directly using the same methodology as in the frequency diversity part.For the 2 receivers that are communicating with the same transmitter, $\Delta D_{T_{j, q}}=0$. Therefore, by combining (12), (13) and (16) for horizontally placed antennas $\left(d_{R_{x}}=0\right)$, the covariance expression for the space-frequency diversity is found as

$$
\begin{aligned}
& E\left[\widehat{\mathbf{h}}_{i, j} \widehat{\mathbf{h}}_{l, q}^{*}\right]=\int_{0}^{r^{10 d B}} \int_{0}^{2 \pi} \zeta(r, \theta) B_{n}(|r \sin (\theta \mid),| r \cos (\theta) \mid)^{2} \\
& \exp \left\{-j 2 \pi\left(\frac{\Delta f}{c}\left(2 R_{r}-r \cos (\theta-\alpha)+r \cos (\pi-\theta-\beta)\right)\right)\right\} \\
& \xi(r, \theta) G_{t}(r, \theta) G_{r}(r, \theta) \exp \left(-j 2 \pi / \lambda d_{R_{y}} r / R_{t} \sin (\theta)\right) d \theta d r .
\end{aligned}
$$

(18) is calculated with numerical integration on MATLAB.

Figure 6 shows the fading correlation for space-frequency diversity for $250 \mathrm{~km}$ range, $50 \mathrm{~m}$ vertical correlation distance and $1.5^{\circ} 3 \mathrm{~dB}$ beam-width. As noticed, the required frequency separation for low correlation decreases with the increasing spatial separation. Similarly, the required spatial separation can be decreased with the frequency separation. For this reason, this employment is especially promising for systems that require placement of multiple antennas in close distances by using both parameters to achieve higher gains in MIMO.

\section{F. Angle Diversity}

Vertical angle diversity is also a promising method for the troposcatter communications because $2 \times 2$ vertical angle diversity system can form four different troposcatter common

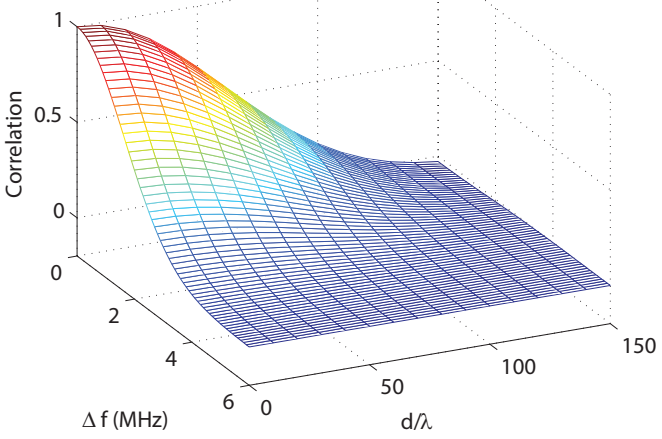

Fig. 6. Fading correlation vs. spatial and frequency separations.

volumes. In addition, the angle diversity receivers can be mounted on the same parabolic reflector. Therefore, the cost of additional antennas will be low compared to space diversity. When the angle spacing of the antennas are higher than the beam-width of the antennas, the troposcatter common volumes will not intersect, and there will be low correlation between the troposcatter common volumes. However, in practical employments, troposcatter systems use very low angular separations because higher angular separation causes increase in the path lengths, and results in more path loss. In angle diversity, the source of the correlation between antennas is the intersection of two different troposcatter common volumes as in Figure 7.

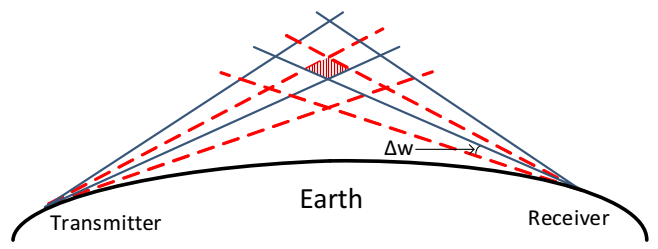

Fig. 7. Troposcatter common volumes in the angle diversity system.

In RSM method, scatterers are assumed to be located just within the ring to make simple theoretical analysis. However, angle diversity system can be analyzed without this assumption with slight modification of the proposed model. For the angle diversity, the shape of the common volume is modeled as parallelogram as in Figure 7. The only source of correlation 
is the intersection of the lower and upper common volumes. The rays that are associated with the intersection point have the same path lengths because the receivers are mounted to the same parabolic reflector, so that $\left(\Delta D_{T_{j, q}}+\Delta D_{R_{i, l}}\right)=0$. Therefore, the correlation for the angle diversity is given as

$$
\begin{array}{r}
E\left[\widehat{\mathbf{h}}_{i, j} \widehat{\mathbf{h}}_{l, q}^{*}\right]=\int_{\alpha_{0}}^{\alpha_{0}+w_{t}^{10 d B}} \int_{\beta_{0}}^{\beta_{0}+w_{r}^{10 d B}+\Delta w} \\
G_{t}^{A}\left(\alpha-\alpha_{0}-w_{t}^{3 d B}\right) G_{r}^{A}\left(\beta-\beta_{0}-w_{r}^{3 d B}-\Delta w\right) \\
B_{n}\left(\left|R_{t} \cos (\alpha)-R / 2\right|,\left|h-h_{C}\right|\right)^{2} d \alpha d \beta,
\end{array}
$$

where $\Delta w$ is the angular spacing, $G_{t, r}^{A}$ are the Gaussian antenna gain functions which is given as $G_{t, r}^{A}(\Theta)=$ $\exp \left(-\Theta^{2} /\left(0.6 \times w^{3 d B}\right)^{2}\right)$ [15]. The middle of the scattering volume is assumed as the reference point for the correlation function $B_{n}$. The scattering height $h$ and the center height of the troposcatter common volume $h_{C}$ is given as [14]

$$
\begin{aligned}
h & =R \frac{\sin (\beta) \sin (\alpha)}{\sin (\alpha+\beta)}, \\
h_{C} & =R \frac{\sin \left(\beta_{0}+w_{t}^{3 d B} / 2\right) \sin \left(\alpha_{0}+w_{t}^{3 d B} / 2\right)}{\sin \left(\alpha_{0}+\beta_{0}+w_{t}^{33 B} / 2+w_{r}^{3 d B} / 2\right)} .
\end{aligned}
$$

Figure 8 shows the angular fading correlation for different beam-widths for $250 \mathrm{~km}$ and $50 \mathrm{~m}$ vertical correlation values. As noticed, the fading correlation becomes very low when the angular separation is higher than the $3 \mathrm{~dB}$ beam-widths, as also suggested in [2]. Since the intersecting region will be higher for higher beam-widths, the correlation for the higher beamwidths are higher in the same separation values. The similar results can be found in [8] for lower $3 \mathrm{~dB}$ beam-widths.

Since increase in the angle causes increase in the path lengths and scattering angle, the upper beam will have considerably higher path-loss. This path-loss difference between lower and upper common volumes is called as squint-loss. The results for the squint-loss are presented in Section VI-A.

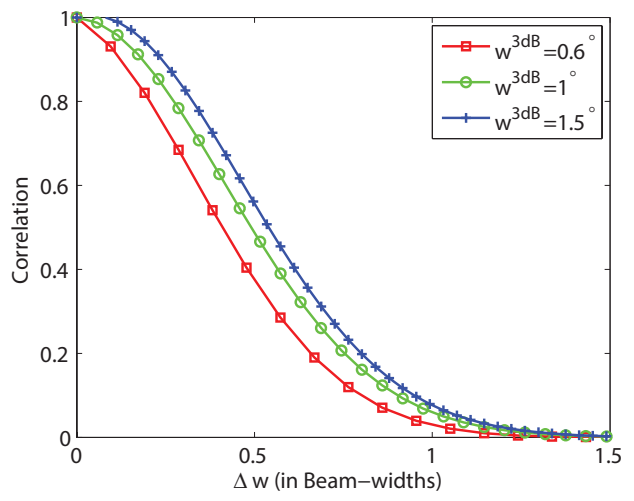

Fig. 8. Fading correlation vs. angular separations.

\section{RAY-BASED TROPOSCATTER CHANNEL MODELING APPROACH}

In this work, we utilize our previously developed ray-based method [1] to compare different diversity techniques in terms of data rate. In addition, we present simulation results for the squint-loss in the angle diversity case. In [1], the beam-width of the antennas are divided into small parts, thus the delay and power calculations for each ray are performed separately. In this way, each ray is subjected to different atmospheric conditions according to its path.

The ray powers are calculated with differential bistatic radar equation

$$
P_{r}=\frac{P_{t} G_{t} G_{r} \sigma_{V} \lambda^{2} \varrho}{(4 \pi)^{3} R_{t}^{2} R_{r}^{2}} \mathrm{~W},
$$

where $P_{t}$ is the transmit power in Watt $(\mathrm{W}), G_{t, r}$ are the antenna gains for transmitter/receiver. $\varrho$ is the polarization mismatch factor. $\sigma_{V}$ is differential scattering cross-section.

The atmospheric profiles are included via the scattering cross-section. We define the differential scattering crosssection by using Rayleigh scattering and Kolmogorov spectrum [16]. In this way, our model can take the real world water-vapor mixing ratio measurements [17] as input to model the non-homogeneities and fluctuations in the air turbulence. The differential scattering cross section is represented as [1]

$$
\sigma_{V}=2 \pi k^{4} \cos (\Psi)^{2} d V_{c} \Phi\left(k_{s}\right) \mathrm{m}^{2},
$$

where $k$ is the wave-number, $\Psi$ is the scatter angle, $d V_{c}$ is the differential scattering volume. In addition, $\Phi\left(k_{s}\right)$ is the Kolmogorov spectrum which is formulated as [16]

$$
\Phi\left(k_{s}\right)=0.33 \pi^{3} C_{n}^{2}(2 k \sin (\Psi / 2))^{-11 / 3} \mathrm{~m}^{3},
$$

where $k_{s}=2 k \sin (\Psi / 2) . C_{n}$ is the structure constant of the refractive-index which is directly generated by using the watervapor mixing ratio measurements [17] (For more details [1]).

\section{Maximum Date Rate}

In this section, we present the calculations for the achievable data rates that can be provided by the b-LoS troposcatter communication under the employment of different diversity techniques. To this end, we improve our ray-based channel modeling approach [1] to implement the diversity techniques in the troposcatter channel with MIMO-OFDM.

\section{A. System Model for Maximum Date Rate}

The block diagram of MIMO-OFDM troposcatter system is in Figure 9. In this work, only single-user to single-user link is considered to show the performance of the troposcatter channel, and the channel is assumed to have slow fading. Full CSI is assumed at both sides of the communication. Therefore, the maximum ratio transmission technique [18] is utilized. In this technique, the transmitter beam-forming is implemented with maximum-ratio-combining (MRC). Transmitter beamforming with MRC reception is also called as MIMO-MRC.

In frequency domain, the $M_{r} \times 1$ received signal vector for $k^{\text {th }}$ OFDM tone $(\mathbf{y}[k])$ is given as

$$
\mathbf{y}[k]=\mathbf{H}\left(e^{j 2 \pi k / N}\right) \mathbf{w}_{t} s+\mathbf{n},
$$

where $s$ is the transmitted symbol with average power $P_{s}, \mathbf{w}_{t}$ is the transmitter beam-forming vector, $\mathbf{n}$ represents AWGN vector, and $N$ is the number of OFDM tones. 


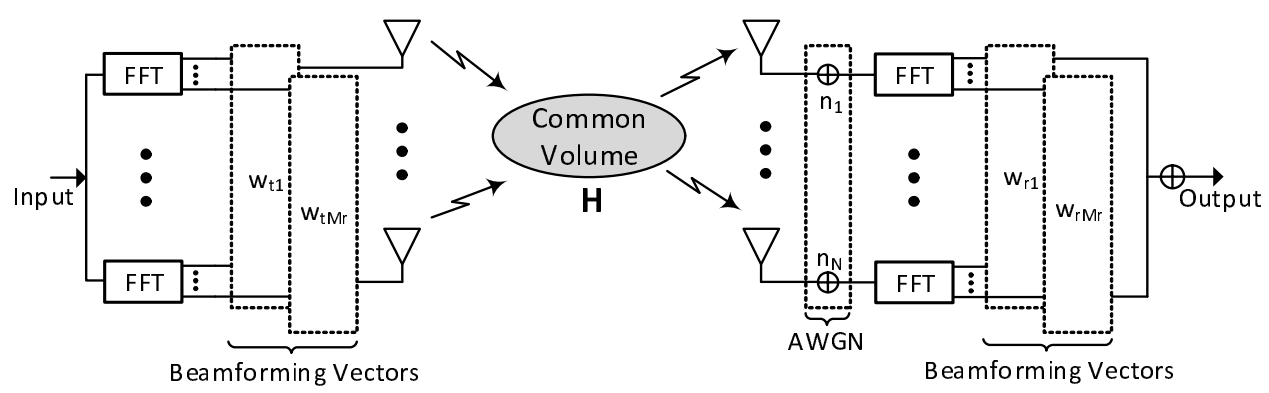

Fig. 9. OFDM antenna array block diagram.

The estimated output signal $(\tilde{\mathbf{y}}[k])$ is given by

$$
\tilde{\mathbf{y}}[k]=\mathbf{w}_{r}^{H} \mathbf{H}\left(e^{j 2 \pi k / N}\right) \mathbf{w}_{t} s+\mathbf{w}_{r}^{H} \mathbf{n},
$$

where (.) ${ }^{H}$ represents the conjugate transpose operation, $\mathbf{w}_{r}$ is the receiver beam-forming vector.

By using the following property $\left\|\mathbf{w}_{r}\right\|^{2}=1$, the SNR for $k^{\text {th }}$ tone can be found with the following expression [19]

$$
\begin{aligned}
\gamma[k] & =\bar{\gamma} \frac{\mathbf{w}_{t}^{H} \mathbf{H}\left(e^{j 2 \pi k / N}\right)^{H} \mathbf{w}_{r} \mathbf{w}_{r}^{H} \mathbf{H}\left(e^{j 2 \pi k / N}\right) \mathbf{w}_{t}}{\mathbf{w}_{r}^{H} \mathbf{w}_{r}} \\
& =\bar{\gamma} \mathbf{w}_{t}^{H} \mathbf{H}\left(e^{j 2 \pi k / N}\right)^{H} \mathbf{H}\left(e^{j 2 \pi k / N}\right) \mathbf{w}_{t},
\end{aligned}
$$

where $\bar{\gamma}=P_{s} / N_{0}$ is the average SNR per receive antenna.

Since we have the $\left\|\mathbf{w}_{t}\right\|^{2}=1$ constraint for the transmit beam-forming vector, $\mathbf{w}_{t}^{H} \mathbf{H}\left(e^{j 2 \pi k / N}\right)^{H} \mathbf{H}\left(e^{j 2 \pi k / N}\right) \mathbf{w}_{t}$ is on the unit sphere. This expression is maximized by the $\lambda_{\max }$, the largest eigenvalue of $\mathbf{H}\left(e^{j 2 \pi k / N}\right)^{H} \mathbf{H}\left(e^{j 2 \pi k / N}\right.$ ) (For more detail, see [18]-[20]). Therefore, the maximum achievable data rate, capacity, is given by

$$
C=\max _{P_{k}: \sum P_{k}=P_{t}} \sum_{k=0}^{N-1} B_{N} \log \left(1+\frac{\lambda_{\max }^{k} P_{k}}{\sigma_{n}^{2} B_{N}}\right),
$$

where $P_{t}$ is the total power constraint, $B_{N}$ is the bandwidth of the each sub-channel $(B / N), \lambda_{\max }^{k}$ is the largest eigenvalue for $\mathbf{H}\left(e^{j 2 \pi k / N}\right)^{H} \mathbf{H}\left(e^{j 2 \pi k / N}\right) . \sigma_{n}^{2}$ is the noise power in $\mathrm{W} / \mathrm{Hz}$ which is modeled as thermal noise. In addition, $P_{k}$ is the allocated power for $k^{\text {th }}$ OFDM sub-channel and they are calculated by the well-known water-filling algorithm [21]

$$
\frac{P_{k}}{P_{t}}= \begin{cases}1 / \gamma_{0}-1 / \gamma_{k}, & \gamma_{k} \geq \gamma_{0} \\ 0, & \gamma_{k}<\gamma_{0}\end{cases}
$$

where $\gamma_{0}$ is the cutoff value and $\gamma_{k}=\lambda_{\max }^{k} P_{t} / \sigma_{n}^{2} B_{N}$. By using (28) and (29), the capacity is simplified into [21]

$$
C=\sum_{k: \gamma_{k} \geq \gamma_{0}} B_{N} \log \left(\gamma_{k} / \gamma_{0}\right)
$$

Up to now, the framework for the capacity calculations is formed. The remaining of the section includes how to generate $\mathbf{H}\left(e^{j 2 \pi k / N}\right)$ matrix with our previously proposed ray-based method and RSM for fading correlation.

\section{B. Channel Gain Matrix for Diversity Techniques}

To generate $\mathbf{H}\left(e^{j 2 \pi k / N}\right)$, we first use the channel matrix normalization to exclude the effects of large-scale path loss which is calculated by the ray-based technique [1]. The largescale path loss is derived by using real world measurements as described in Section IV. The small-scale fading is assumed as Rayleigh fading as suggested in [3], [7], [15], [22], [23]. Normalized channel gain matrix $\tilde{\mathbf{H}}\left(e^{j 2 \pi k / N}\right)$ is found as [24]

$$
\tilde{\mathbf{H}}\left(e^{j 2 \pi k / N}\right)=\frac{\mathbf{H}\left(e^{j 2 \pi k / N}\right)}{\sqrt{\frac{1}{M_{t} M_{r}}\left\|\mathbf{H}\left(e^{j 2 \pi k / N}\right)\right\|_{F}^{2}}},
$$

where $\|.\|_{F}$ is the Frobenius norm. Thus, the average SNR on the channel is found as [24], [25]

$$
S N R_{a v}=P_{t} /\left(\sigma_{n}^{2} M_{t} M_{r}\right)\left\|\mathbf{H}\left(e^{j 2 \pi k / N}\right)\right\|_{F}^{2},
$$

where $S N R_{a v}$ is the average receive SNR that is calculated with the ray-based method. As described in Section IV, the water-vapor mixing ratio measurements are utilized for atmospheric modeling, and the data set includes 1000 measurements that are measured in August near Cape Verde Islands [17]. Therefore, the average SNR is calculated for each measurement instant to model the atmospheric fluctuations.

With the channel normalization, (27) can be represented as

$$
\begin{array}{r}
\mathbf{w}_{t}^{H} \mathbf{H}\left(e^{j 2 \pi k / N}\right)^{H} \mathbf{H}\left(e^{j 2 \pi k / N}\right) \mathbf{w}_{t}= \\
S N R_{a v} \mathbf{w}_{t}^{H} \tilde{\mathbf{H}}\left(e^{j 2 \pi k / N}\right)^{H} \tilde{\mathbf{H}}\left(e^{j 2 \pi k / N}\right) \mathbf{w}_{t} .
\end{array}
$$

This expression is maximized by the largest eigenvalue of $\tilde{\mathbf{H}}\left(e^{j 2 \pi k / N}\right)^{H} \tilde{\mathbf{H}}\left(e^{j 2 \pi k / N}\right)$, and $\tilde{\mathbf{H}}$ is generated for each diversity technique differently.

1) Space diversity: With the correlation results that are generated in Section III-C, the normalized channel gain matrix is modeled as [26]

$$
\tilde{\mathbf{H}}\left(e^{j 2 \pi k / N}\right)=\left(\mathbf{R}_{H}^{R X}\right)^{1 / 2} \mathbf{G}\left(\mathbf{R}_{H}^{T X}\right)^{1 / 2},
$$

where $\mathbf{G}$ is the complex Gaussian iid matrix. $\left(\mathbf{R}_{H}^{R X}\right)^{1 / 2}$ is the root of the receiver antenna correlation matrix $\left(\mathbf{R}_{H}^{R X}\right)^{1 / 2}\left[\left(\mathbf{R}_{H}^{R X}\right)^{1 / 2}\right]^{H}=\mathbf{R}_{H}^{R X}$ which is found by Cholesky factorization. $\left(\mathbf{R}_{H}^{T X}\right)$ is the transmitter correlation matrix.

2) Frequency diversity: Since the frequency separation between antennas nulls the cross components, there are $M_{t}=$ $M_{r}$ parallel channels in frequency diversity. Therefore, $\mathbf{G}$ in (34) is generated as diagonal matrix for frequency diversity. 
3) Space-frequency diversity: Since the frequency diversity is provided with only one parabolic reflector, we can use horizontally placed antennas with frequency diversity, and achieve quad-diversity. Suppose that $N_{S F D}$ represents the number of parabolic reflectors in one side and there are equal number of them in each side, $\mathbf{G}$ matrix in (34) for the spacefrequency diversity becomes

$$
\mathbf{G}_{M_{F} N_{S F D} \times M_{F} N_{S F D}}=\operatorname{diag}\left(\mathbf{G}_{1}, \ldots, \mathbf{G}_{N_{S F D}}\right) .
$$

where $\mathbf{G}_{1, \ldots, N_{S F D}}$ represents the complex Gaussian iid channel matrix with $M_{F} \times M_{F}$, where $M_{F}$ is the number of frequency used for the frequency diversity. The function diag forms a block diagonal matrix from its input arguments.

4) Angle diversity: In angle diversity, the increase in the elevation angles cause significant decrease in the average SNR. Instead of channel matrix normalization, the average SNR for each antenna pair is calculated separately for $M_{t}=M_{r}$ case. (27) is represented as (36) for $M_{t}=M_{r}$ angle diversity system where $\circ$ is Hadamard product that is element by element matrix multiplication. Therefore, (36) is maximized with the largest eigenvalue of $\left[S N R_{a v i, j}\right]_{i=1}=\ldots, M_{r} \circ$ $\tilde{\mathbf{H}}\left(e^{j 2 \pi k / N}\right)^{H} \tilde{\mathbf{H}}\left(e^{j 2 \pi k / N}\right)$. (36) is calculated with the raybased technique for each antenna pair for the angle diversity. $\tilde{\mathbf{H}}\left(e^{j 2 \pi k / N}\right)$ term in (36) is also modeled as in (34).

\section{Vi. Performance Evaluation}

In this section, we compare the troposcatter diversity techniques by using maximum data rate simulations. In addition, we present the squint-loss results for the angle diversity.

\section{A. Squint-Loss Results}

For space and frequency diversity cases, the antennas utilize the same vertical orientation. Therefore, different antenna pairs in space and frequency diversity systems can maintain the same average received power levels. On the other hand, the angle diversity utilize angular separation and upper beam is subjected to significantly higher path-loss because both the path lengths and the scattering angle increase. For this reason, we analyze the additional path-loss caused by the angular separation which is also known as squint-loss.

We utilize the ray-based channel model [1] as in Section IV for the following channel parameters: $250 \mathrm{~km}, 4.7 \mathrm{GHz}$ carrier frequency, $41.5 \mathrm{~dB}$ antenna gains in each side. Figure 10 includes the squint-loss results for different beam-widths. As noticed, the squint loss increases with increasing beam-width because the separations are given in beam-widths. Similar results for the squint-loss is presented in [9] for the angle diversity. According to the experimental results, the upper and

$$
\mathbf{w}_{t}^{H} \mathbf{H}\left(e^{j 2 \pi k / N}\right)^{H} \mathbf{H}\left(e^{j 2 \pi k / N}\right) \mathbf{w}_{t}=\frac{1}{M_{t}} \mathbf{w}_{t}^{H}\left[\begin{array}{ccc}
S N R_{a v 1,1} & \cdots & S N R_{a v 1, M_{t}} \\
\vdots & \ddots & \vdots \\
S N R_{a v M_{r}, 1} & \cdots & S N R_{a v M_{r}, M_{t}}
\end{array}\right] \circ\left(\tilde{\mathbf{H}}\left(e^{j 2 \pi k / N}\right)^{H} \tilde{\mathbf{H}}\left(e^{j 2 \pi k / N}\right)\right) \mathbf{w}_{t} .
$$

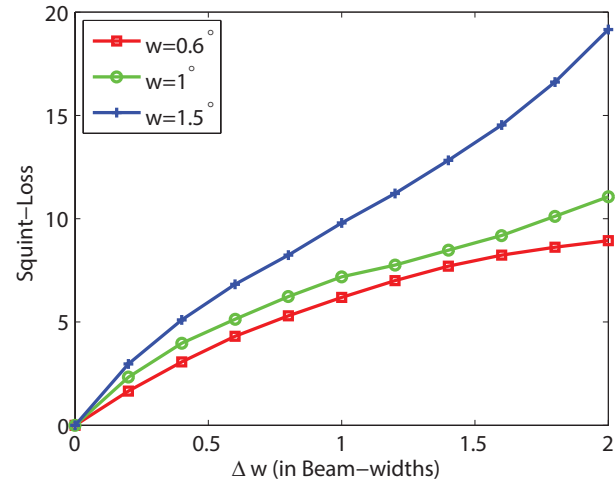

Fig. 10. Squint-loss results for angle diversity.

lower beams show $5-10 \mathrm{~dB}$ squint-loss for $0.6^{\circ} 3 \mathrm{~dB}$ beamwidth and $1^{\circ}$ beam separation ( $\Delta w \approx 1.6$ in beam-width). As noticed in Figure 10, the average squint-loss calculated with [1] is also in the experimentally measured range.

\section{B. Maximum Data Rate Results}

We present the achievable data rate simulation results by using the RSM to estimate the required separations for the partial correlation between antennas. The required separations for partial correlations (for $\rho=0.5$ ) can be found in Table I.

TABLE I

TROPOSCATTER DIVERSITY SEPARATIONS.

\begin{tabular}{lcc}
\hline Diversity technique & Separations & Correlation $(\rho)$ \\
\hline \hline Space diversity $(2 \times 2)$ & $d=65 \lambda \mathrm{m}$ & 0.5 \\
\hline Frequency diversity $(2 \times 2)$ & $\Delta f=2 \mathrm{MHz}$ & 0.5 \\
\hline Angle diversity $(2 \times 2)$ & $\begin{array}{c}\Delta w=14 \mathrm{mrad} \\
\text { Squint-loss }=6 \mathrm{~dB}\end{array}$ & 0.5 \\
\hline Space-frequency diversity $(4 \times 4)$ & $\begin{array}{c}d=30 \lambda \mathrm{m} \\
\Delta f=1.7 \mathrm{MHz}\end{array}$ \\
\hline
\end{tabular}

The troposcatter simulation parameters can be found in Table II. In addition, there are 1000 atmospheric measurements in the used data sets [17]. We perform 1000 realization for the random $\mathbf{G}$ matrix as in Section V-B. Therefore, the maximum data rate results are generated with the total of 1 million realization by using the ray-based model [1] in MATLAB.

Figure 11 presents the cumulative distribution function (CDF) of the data rate for different diversity techniques. As expected, $2 \times 2$ space diversity provides significantly higher data rates compared to frequency and angle diversity. In addition, the span of achievable data rates decreases with the space diversity, thus the additional antenna makes the channel more reliable. However, $2 \times 2$ frequency diversity provides the lowest data rate gains because the frequency 


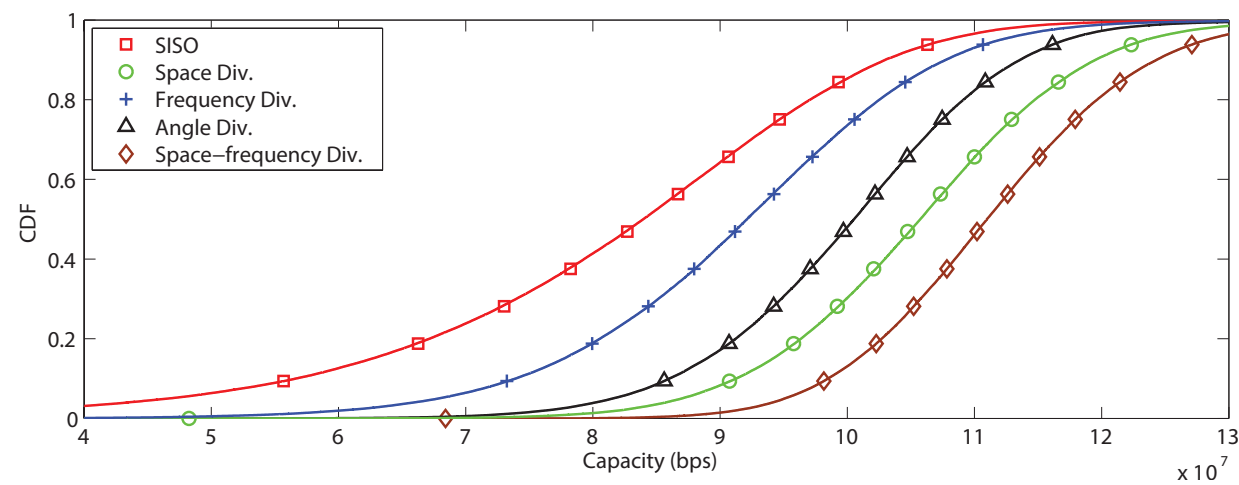

Fig. 11. The distribution of the achievable data rates for different diversity techniques.

TABLE II

TROPOSCATTER COMMUNICATION PARAMETERS.

\begin{tabular}{lcc}
\hline Parameter & Value & Unit \\
\hline \hline Horizontal distance $(d)$ & 250 & $\mathrm{~km}$ \\
\hline Carrier frequency $(f)$ & 4.7 & $\mathrm{GHz}$ \\
\hline Transmit power $\left(P_{t}\right)$ & 1000 & $\mathrm{~W}$ \\
\hline Bandwidth $(B)$ & 10 & $\mathrm{MHz}$ \\
\hline Average Transmission-loss $\left(P_{r} / P_{t}\right)$ & -138 & $\mathrm{~dB}$ \\
\hline \# of OFDM symbols $(N)$ & 64 & - \\
\hline Beam-widths $\left(w_{t, r}\right)$ & 26 & $\mathrm{mrad}$ \\
\hline Beam-elevations $\left(\alpha_{0}, \beta_{0}\right)$ & 14.7 & $\mathrm{mrad}$ \\
\hline Antenna gains $\left(G_{t, r}\right)$ & 41.5 & $\mathrm{dBi}$ \\
\hline Antenna diameters $\left(D_{\text {ant }}\right)$ & 3 & $\mathrm{~m}$ \\
\hline
\end{tabular}

separation between antennas nulls the cross antenna signal powers. On the other hand, $2 \times 2$ angle diversity, which can be performed with only 2 parabolic reflectors, has significantly higher performance compared to the frequency diversity. Since the increase in the elevation angle decreases the received power of the additional troposcatter common volumes, the space diversity outperforms the angle diversity.

Although the frequency diversity has the worst achievable data rate performance, the space-frequency diversity can provide quad diversity by using 2 parabolic reflectors in each side. In this case, there are 2 horizontally placed antennas, and each antenna mounted with $2 \times 2$ frequency diversity receivers. As seen in Figure 11, the achievable data rate performance of the space-frequency diversity is higher than the space diversity, and its implementation is much more economical compared to adding additional parabolic reflector antennas.

\section{CONCLUSION}

In this paper, RSM for fading correlation is proposed to investigate the fading correlation between antennas for space, frequency, angle and space-frequency diversity. In addition, we compare these diversity techniques with their distribution of achievable data rates. According to our results, spacefrequency diversity systems can provide more than $10 \%$ increase in the achievable data rates. Since the implementation of frequency diversity does not require additional parabolic reflector, space-frequency diversity systems are much more economical compared to adding additional antennas. Furthermore, the simulation results shows that the high powered b-LoS troposcatter communication can provide more than $8 \mathrm{Mbps}$ at all times. Therefore, the troposcatter communication is a promising candidate for high data rate b-LoS communications with the employment of diversity techniques.

\section{APPENDIX A \\ INTEGRAL BOUNDS}

In (8), the integral bound is given as the whole ring defined by the $10 \mathrm{~dB}$ beam-width. However, the lower part of the ring should be excluded from the integral as also shown in Figure 12. To this end, we define a function $\zeta(r, \theta)$ to exclude this area from the integral, and it is given as

$$
\zeta(r, \theta)= \begin{cases}0, & \text { if } r>r^{10 d B} \cos \left(\pi / 2-\theta+\alpha_{0}\right), \text { and } \\ & \pi-\alpha_{0}+\varphi>\theta>\alpha_{0}+\varphi \\ 1, & \text { otherwise }\end{cases}
$$

where $\varphi=\arcsin \left(r^{3 d B} / r^{10 d B}\right)$.

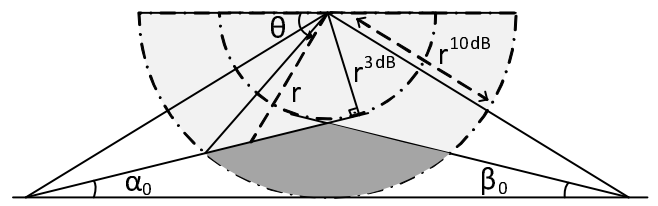

Fig. 12. The integral boundaries.

\section{APPENDIX B \\ ANTENNA GAINS}

Since RSM model utilize the $10 \mathrm{~dB}$ beam-width of the antennas, we include the antenna gains in the correlation calculations. To this end, we utilize Gaussian antenna pattern [15]. For the RSM method, we also need to represent the antenna gains as a function of $r$ and $\theta$. To this end, we utilize Figure 2 and geometrical approximations that are introduced in Section III-C, thus the antenna gains is represented as

$$
G_{t}(r, \theta)=e^{\left(-\frac{\left(\alpha_{0}+\frac{w_{t}^{3 d B}}{2}-\arctan \left(\frac{r \sin (\theta)}{R_{t}-r \cos (\theta)}\right)\right)^{2}}{\left(0.6 \times w_{t}^{3 d B}\right)^{2}}\right)}
$$




$$
G_{r}(r, \theta)=e^{\left(-\frac{\left(\beta_{0}+\frac{w_{t}^{3 d B}}{2}-\arctan \left(\frac{r \sin (\pi-\theta)}{R_{t}-r \cos (\pi-\theta)}\right)\right)^{2}}{\left(0.6 \times w_{t}^{3 d B}\right)^{2}}\right)}
$$

\section{APPENDIX C $\xi(r, \theta)$ FUNCTION}

To simplify the correlation equations, we define $\xi(r, \theta)$ to represent the sine multiplication, and this function is given as

$$
\begin{aligned}
\xi(r, \theta) & =\sin \left(\Psi_{1}\right)^{-\frac{11}{3}} \sin \left(\Psi_{2}\right)^{-\frac{11}{3}} \\
& =\left(\frac{\cos \left(\frac{\Psi_{1}+\Psi_{2}}{2}\right)-\cos \left(\frac{\Psi_{1}-\Psi_{2}}{2}\right)}{2}\right)^{-\frac{11}{3}},
\end{aligned}
$$

Since the separation between antennas is very small in the troposcater communication between $70-100 \lambda$, there will be a small difference between the scattering angles for the different antenna pairs in the space diversity. In frequency and angle diversity, the antennas are mounted on the same parabolic reflector therefore, the scattering angles will be same therefore, we assume $\Psi_{1}=\Psi_{2}$. Thus, the $\xi$ can be further simplified as

$$
\xi(r, \theta)=\left[\left(\cos \left(\Psi_{1} / 2+\Psi_{2} / 2\right)-1\right) / 2\right]^{(-11 / 3)} .
$$

The scattering angles are calculated with the following equation for both of the antennas,

$$
\begin{aligned}
\Psi(r, \theta)= & \arctan \left(\frac{h-r \sin (\theta)}{d / 2-r \cos (\theta)}\right)+ \\
& +\arctan \left(\frac{h-r \sin (\theta)}{d / 2+r \cos (\theta)}\right) .
\end{aligned}
$$

\section{REFERENCES}

[1] E. Dinc, O. B. Akan, "A Ray-based Channel Model For MIMO Troposcatter Communications," Proc. IEEE PIMRC, London, UK, Sept. 2013.

[2] Comtech Systems, Technical Information, "Introduction to Troposcatter Communications: a brief synopsis of over-the-horizon troposcatter" [Online]. Available: http://www.comtechsystems.com/wpcontent/uploads/2014/05/Troposcatter-Introduction-Nov-2013.pdf (URL).

[3] "ITU-R's Rec. P. 617-2: Propagation prediction techniques and data required for the design of trans-horizon radio-relay systems," ITU, 2012.

[4] W. S. Patrick, "Troposcatter for Tactical Communications," IEEE Trans. Mil. Electron., vol. 9, no. 2, pp. 137-143, Apr. 1965.

[5] D. Kennedy, "A Comparison of Measure and Calculated Frequency Correlation Functions over 4.6 and 7.6-GHz Troposcatter Paths," IEEE Trans. Commun., vol. 20, no. 2, pp. 173-178, Apr. 1972.

[6] G. C. Rider, "Some tropospheric scatter propagation measurements and tests of aerial siting conditions at $858 \mathrm{Mc} / \mathrm{s}$," Proceedings of the IEE, vol. 105 , no. 8, pp. 143-152, 1958.

[7] R. W. Meadows, "Tropospheric scatter observations at $3480 \mathrm{Mc} / \mathrm{s}$ with aerials of variable spacing," Proceedings of the IEE, vol. 108, iss. 40, p. 349-360, July 1961.

[8] M. W. Gough, G. C. Rider, "Angle diversity in troposcatter communications. Some confirmatory trials," Proceedings of the IEE, vol. 122, no. 7, pp. 713-719, July 1975.

[9] P. Monsen, "Performance of an Experimental Angle-Diversity Troposcatter System," IEEE Trans. Commun., vol. 20, pp. 242-247, Apr. 1972.

[10] S. B. Rhee and G. I. Zysman, "Results of suburban base-station spatial diversity measurements on the UHF band," IEEE Trans. Commun., pp. 1630-1634, Oct. 1974

[11] Shiu Da-shan, G. J. Foschini, M. J. Gans, J. M. Kahn, "Fading correlation and its effect on the capacity of multielement antenna systems," IEEE Trans. Commun., vol. 48, no. 3, pp. 502-513, Mar. 2000.

[12] L. Bastos, H. Wietgrefe, "Tactical troposcatter applications in challenging climate zones," Proc. IEEE MILCOM 2012, pp. 1-6, 2012.

[13] W. C. Jakes, Microwave Mobile Communications. New York: Wiley, pp. 6065,1974

[14] G. Roda, Troposcatter Radio Links. Artech House Publishers, 1988.
[15] P. Bello, "A Troposcatter Channel Model," IEEE Trans. Commun. Technol., vol. 17, no. 2, pp. 130-137, April 1969.

[16] A. Ishimaru, Wave Propagation and Scattering in Random Media. IEEE Press Series on Electromagnetic Wave Theory, Wiley, 1999.

[17] NAMMA LIDAR Atmospheric Sensing Experiment (LASE), NASA EOSDIS GHRC DAAC, [Online]. Available: http://ghrc.msfc.nasa.gov/index.html. Accessed on Jan 30, 2013.

[18] T. K. Y. Lo, "Maximum ratio transmission," Proc. IEEE ICC, vol. 2, pp. 1310-1314 vol. 2, 1999

[19] A. Maaref, S. Aissa, "Closed-form expressions for the outage and ergodic Shannon capacity of MIMO MRC systems," IEEE Trans. Commun., vol. 53, no. 7, pp. 1092-1095, July 2005.

[20] K. Ahn, "Performance analysis of MIMO-MRC system in the presence of multiple interferers and noise over rayleigh fading channels," IEEE Trans. Wireless Commun., vol. 8, no. 7, pp. 3727-3735, July 2009.

[21] A. Goldsmith, Wireless Communications, Cambridge University Press, 2005.

[22] Y. Zhao, X. Chen, "Research on MRC based on Rake receiver in troposcatter communication," Proc. CECNet, pp. 4342-4345, 2011

[23] Y. Wang, Y. Fang, X. Da, W. Jin, "Study on Modeling of Troposcatter Communication and MRC in Correlated Channel by Matlab," Proc. WiCOM, pp. 1-4, 2008.

[24] P. Kyritsi, D. C. Cox, "Effect of element polarization on the capacity of a MIMO system," Proc. IEEE WCNC, vol. 2, pp. 892-896, Mar. 2002

[25] Kai Yu, M. Bengtsson, B. Ottersten, D. McNamara, P. Karlsson, M. Beach, "Second order statistics of NLOS indoor MIMO channels based on 5.2 GHz measurements," Proc. IEEE GLOBECOM, pp. 156-160, 2001.

[26] D. Gesbert, H. Bolcskei, D. Gore, A. Paulraj, "MIMO wireless channels: capacity and performance prediction," Proc. IEEE GLOBECOM, vol. 2, pp. 1083-1088, 2000

[27] A. Ishimaru, "Theory and application of wave propagation and scattering in random media," Proc. IEEE, vol. 65, no. 7, pp. 1030-1061, July 1977.

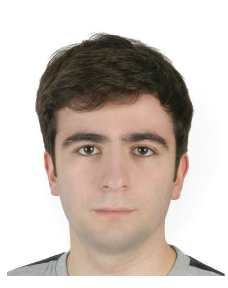

Ergin Dinc [S'12] (edinc@ku.edu.tr) received his B.Sc. degree in Electrical and Electronics Engineering from Bogazici University, Istanbul, Turkey, in 2012. He is currently a research assistant at NextGeneration and Wireless Communications Laboratory (NWCL) and pursuing his Ph.D. degree at the Electrical and Electronics Engineering Department, Koc University, Istanbul, Turkey. His current research interests include communication theory, beyond-Line-of-Sight (b-LoS) communications with troposcatter and atmospheric ducts.

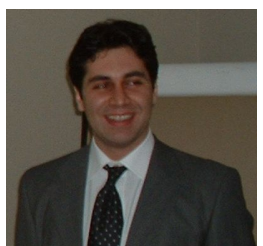

Ozgur B. Akan [M00-SM07] (akan@ku.edu.tr) received his Ph.D. degree in electrical and computer engineering from the Broadband and Wireless Networking Laboratory, School of Electrical and Computer Engineering, Georgia Institute of Technology in 2004. He is currently a full professor with the Department of Electrical and Electronics Engineering, Koc University and the director of the Next-generation and Wireless Communications Laboratory. His current research interests are in wireless communications, nano-scale and molecular communications, and information theory. He is an Associate Editor of the IEEE Transactions on Communications, the IEEE Transactions on Vehicular Technology, the International Journal of Communication Systems (Wiley), the Nano Communication Networks Journal (Elsevier), and the European Transactions on Technology. 